УДК 332.14

ББК 65.04

МЕТОДИЧЕСКОЕ ОБЕСПЕЧЕНИЕ ОЦЕНКИ РЕЗУЛЬТАТИВНОСТИ РЕАЛИЗАЦИИ СТРАТЕГИИ РАЗВИТИЯ ГОРОДА И ЕЕ КОРРЕКТИРОВКИ

\author{
С. А. БУНЬКО \\ swetlanabunko@mail.ru
}

старший преподаватель кафедры мировой экономики, маркетинга, инвестиций

Брестский государственный технический университет

Брест, Республика Беларусь

Стратегия развития города рассматривается как основная форма управления конкурентоспособностью города. Предлагается методическое обеспечение оценки результативности реализации стратегии города и ее корректировки с учетом динамики конкурентоспособности, включающее: алгоритм оценки результативности стратегии, методику оценки степени выполнения показателей с учетом коэффициентов важности и блок-схему прочесса принятия решения о корректировке стратегии/стратегического плана.

Ключевые слова: конкурентоспособность, город, стратегия развития, оценка результативности, корректировка.

\title{
METHODOLOGICAL SUPPORT OF ASSESSMENT OF EFFECTIVENESS OF IMPLEMENTATION OF THE STRATEGY OF DEVELOPMENT FOR THE CITY AND ITS ADJUSTMENT
}

\author{
S. A. BUNKO \\ senior lecturer of department of world economy, marketing, investments \\ Brest state technical university \\ Brest, Republic of Belarus
}

The strategy of city development is considered as the main form of management to city competitiveness. The methodological support of assessment of effectiveness of city strategy's realization and its adjustment taking into account dynamics of competitiveness including an algorithm of efficience assessment of strategy, an assessment technique of extent of performance of indicators taking into account coefficients of importance and the flowchart of making decision's process on correction of strategy / the strategic plan.

Keywords: competitiveness, city, development strategy, effectiveness assessment, adjustment.

\section{ВВЕДЕНИЕ}

Признанной формой управления развитием города, составляющей основу планирования повышения конкурентоспособности в различных странах, является стратегия его развития, которую следует рассматривать в двух аспектах: во-первых, как процесс; во-вторых, как форму территориального управления, которая предполагает обязательное наличие документа стратегии. В контексте первого аспекта под стратегией понимается совокупность направлений деятельности и соответствующих решений стратегического характера, которые состоят в разработке мероприятий, направленных на повышение конкурентоспособности города, а также в разработке механизмов и инструментов реализации 
выбранной стратегии [1, с. 19]. В контексте второго аспекта, стратегия - документ, определяющий общие цели, задачи, направления развития города, механизмы их реализации, вытекающие из анализа ресурсных возможностей, существующей социально-экономической ситуации и возможных сценариев развития в зависимости от внешних по отношению к городу условий [2].

К настоящему времени в различных странах накоплен значительный теоретический и практический опыт разработки и реализации стратегий развития. В Беларуси методические рекомендации по разработке региональных стратегий предложены в рамках проекта международной помощи ЕС «Поддержка регионального и местного развития в Беларуси». Однако оценка результативности и процесс принятия решений о корректировке как действий по реализации стратегии, так и самой стратегии недостаточно формализованы. Вместе с тем, эти процессы являются важным этапом управления конкурентоспособностью города, поскольку дают возможность оценить степень выполнения показателей, в случае их невыполнения выявить причины и принять решение о корректирующих действиях как в отношении усилий по реализации стратегии, так и корректировке самой стратегии Целью данной статьи является разработка методического обеспечения оценки результативности реализации стратегии и процесса принятия решений о корректирующих действиях.

\section{РЕЗУЛЬТАТЫ И ИХ ОБСУЖДЕНИЕ}

Сложность управления конкурентоспособностью города предопределяется многочисленными прямыми и обратными связями социально-экономической системы города. Особенность управления состоит в том, что для оценки реализации экономической целесообразности стратегических решений должны использоваться не только и не столько критерии локальной экономической эффективности организаций или отраслей, сколько критерии социальной эффективности. Кроме того, зачастую результаты реализации стратегии имеют отложенный эффект, то есть проявляются не сразу. Еще одна особенность в том, что периодичность статистической отчетности не позволяет осуществлять такие функции текущего управления как организация и контроль процессов, а также оперативное управление. Это затрудняет быстрое применение корректирующих и управляющих воздействий. С учетом этих особенностей нами предлагается алгоритм мониторинга реализации и корректировки стратегии развития, представленный на рисунке 1.

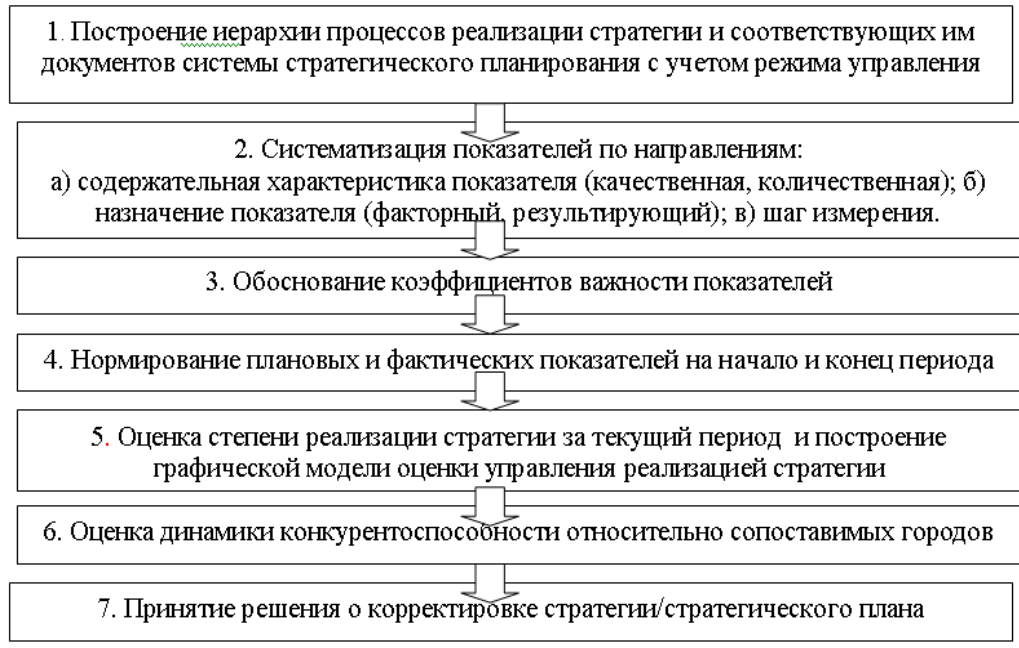

Рисунок 1 - Алгоритм оценки результативности реализации стратегии развития Источник: разработка автора 
Особую трудность вызывает построение иерархической модели процесса реализации стратегии, приведение показателей (как факторных, так и результирующих) в сопоставимый вид, а также определение степени важности тех или иных показателей. Нами предлагается четырехуровневая модель процесса реализации стратегии развития города (рис. 2).

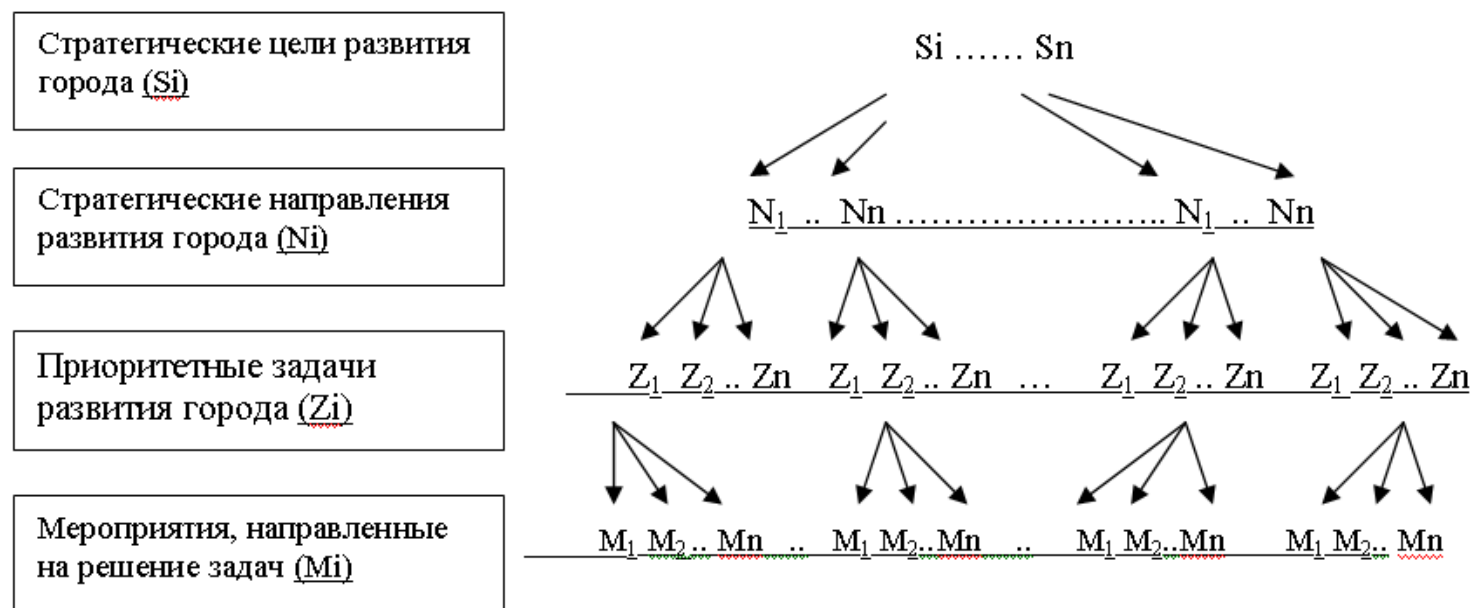

Рисунок 2 - Иерархия процессов реализации стратегии развития города Источник: разработка автора

Построение иерархии процессов реализации стратегии развития города предопределяет иерархическую структуру системы документов стратегического управления с учетом длительности режимов управления (таблица 1).

Таблица 1 - Иерархия системы документов стратегического планирования

\begin{tabular}{|l|l|c|l|}
\hline Режим управления & Вид документа & $\begin{array}{l}\text { Длительность } \\
\text { периода }\end{array}$ & \multicolumn{1}{|c|}{ Направление оценки } \\
\hline $\begin{array}{l}\text { Стратегическое } \\
\text { прогнозирование }\end{array}$ & Стратегия & 20 лет & $\begin{array}{l}\text { Оценка взаимосвязи «степень } \\
\text { реализации стратегических } \\
\text { направлений - степень достижения } \\
\text { стратегических целей» }\end{array}$ \\
\hline $\begin{array}{l}\text { Стратегическое } \\
\text { планирование }\end{array}$ & $\begin{array}{l}\text { Стратегический } \\
\text { план }\end{array}$ & 5 лет & $\begin{array}{l}\text { Оценка взаимосвязи «степени } \\
\text { решения задач - степень реализации } \\
\text { стратегических направлений» }\end{array}$ \\
\hline $\begin{array}{l}\text { Текущее планиро- } \\
\text { вание }\end{array}$ & $\begin{array}{l}\text { План первооче- } \\
\text { редных действий }\end{array}$ & 1 год & $\begin{array}{l}\text { Оценка взаимосвязи «степень } \\
\text { реализации мероприятий - степень } \\
\text { решения задач» }\end{array}$ \\
\hline
\end{tabular}

Источник: разработка автора

Исходя из длительности периодов управления возможно определение интервала измерения и приращения времени между контрольными точками измерения с постоянным шагом $\Delta \mathrm{t}$. Интервал времени между измерениями при управлении устойчивым развитием предприятия, по мнению О. А. Высоцкого, должен вмещать не менее трех контрольных точек воздействия. Такие контрольные точки «необходимы для настройки систем и процессов управления .... и корректировки векторов сил развития для адекватных воздействий на процессы устойчивого развития» [3, с. 15-16]. Применяя это правило к управлению конкурентоспособностью и развитием города, можно выделить интервал времени между измерениями для стратегического прогнозирования и планирования. Для прогнозирования интервал измерений составит 20 лет, для планирования - 5 лет 
(контрольные точки $\mathrm{T}_{\kappa}$ ), для текущего - шаг между контрольными точками $\left(\mathrm{t}_{\mathrm{\kappa}}\right)$ будет равен одному году.

Предлагаемая иерархия предполагает также следующее: нижележащий уровень показателей будет являться по отношению к вышестоящим наборам факторных показателей; каждый вышестоящий уровень по отношению к нижележащему - результирующим. Так, показатели, характеризующие приоритетные задачи развития, будут являться результирующими по отношению к показателям, характеризующим мероприятия, направленные на решение этих задач, и факторными для показателей, характеризующих стратегические направления развития. Оценка взаимосвязи степени реализации стратегических направлений и степени достижения стратегических целей дает возможность оценить качество стратегического прогнозирования, выбора стратегических направлений для достижения задач. В связи с тем, что зачастую эффект является отложенным, оценка целесообразна с интервалом не менее 5 лет. Оценка взаимосвязи степени решения задач и степени реализации стратегических направлений дает возможность оценить правильность поставленных задач. Осуществляется по истечении пятилетнего периода стратегического планирования и предварительно по контрольным точкам с шагом контроля в 1 год. Такая оценка позволит оценить: а) действенность предпринимаемых для решения поставленных задач мер; б) достаточность финансовых средств, выделяемых на решение поставленных задач; в) уровень компетентности лиц, ответственных за реализацию запланированных мероприятий; г) необходимость корректировки.

Для осуществления таких оценок необходимо определить степень выполнения показателей. Кроме того, возникает необходимость разработки коэффициентов их важности. Считаем целесообразным рассчитывать степень выполнения показателей показатели таким образом, чтобы в каждой контрольной точке планируемые показатели равнялись единице, а фактические характеризовали степень реализации запланированной динамики этих показателей. Алгоритм расчета с учетом коэффициентов важности представлен в таблице 2.

Таблица 2 - Методика оценка степени выполнения показателей

\begin{tabular}{|c|c|}
\hline Этап & Формула расчета \\
\hline 1 & 2 \\
\hline $\begin{array}{l}\text { Оценка степени дости- } \\
\text { жения факторных по- } \\
\text { казателей }\end{array}$ & $\begin{array}{l}\text { если фактор приводит к росту конкурентоспособности: } \\
O P_{i}^{j}=\frac{p_{j \text { факт }}^{i}-p_{j \text { нач }}^{i}}{p_{j \text { пл }}^{i}-p_{j \text { нач }}^{i}}, \text { если к снижению: } O P_{i}^{j}=-\frac{p_{j \text { факт }}^{i}-p_{j \text { нач }}^{i}}{p_{j \text { пл }}^{i}-p_{j \text { нач }}^{i}} \\
\text { где } O P_{i}^{j}-\text { оценка степени достижения } \mathrm{j} \text {-го факторного показателя } \\
\text { для достижения і-го результирующего показателя; } p_{j \text { пл }}^{i}, p_{j \text { нач }}^{i}, \\
p_{j}^{i} \text { факт - значения } \mathrm{j}-\text { го факторного показателя: планового и факти- } \\
\text { ческого на момент времени } \mathrm{t}_{\text {к }}, \text { на начало периода измерения }\end{array}$ \\
\hline $\begin{array}{l}\text { Расчет интегрального } \\
\text { индекса выполнения } \\
\text { факторных показате- } \\
\text { лей }\end{array}$ & 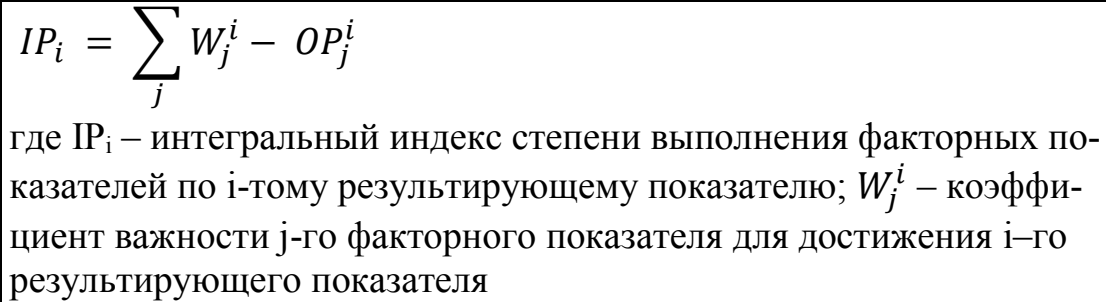 \\
\hline
\end{tabular}


Окончание таблицы 2

\begin{tabular}{|c|c|}
\hline $\begin{array}{l}\text { Оценка степени } \\
\text { выполнения результа- } \\
\text { тивных показателей }\end{array}$ & $\begin{array}{l}\text { если показатель необходимо максимизировать: } \\
O R_{i}=\frac{r_{i \text { факт }}-r_{i \text { нач }}}{r_{i \text { пл }}-r_{i \text { нач }}}, \text { минимизировать: } O R_{i}=-\frac{r_{i \text { факт }}-r_{i \text { нач }}}{r_{i \text { пл }}-r_{i \text { нач }}}, \\
\text { где } O R_{i}-\text { оценка степени достижения і-го результирующего показа- } \\
\text { теля; } r_{i n л}, r_{i \text { нач }}, r_{i} \text { факт - значения і-го результирующего показа- } \\
\text { теля: планового на момент времени } \mathrm{t}_{\text {к }} \text {, на начало периода измере- } \\
\text { ния, фактического на момент времени } \mathrm{t}_{\mathrm{\kappa}}\end{array}$ \\
\hline $\begin{array}{l}\text { Расчет интегрального } \\
\text { индекса выполнения } \\
\text { результативных пока- } \\
\text { зателей }\end{array}$ & $\begin{array}{l}I R_{i}=\sum_{i} \mathrm{~W}_{\mathrm{i}} \cdot \mathrm{OR}_{\mathrm{i}}, \\
\text { где } \mathrm{IR}_{\mathrm{i}}-\text { интегральный индекс степени выполнения і-го результиру- } \\
\text { ющего показателя; } W_{i} \text { - коэффициент важности і-го результирую- } \\
\text { щего показателя }\end{array}$ \\
\hline $\begin{array}{l}\text { Оценка степени } \\
\text { выполнения } \\
\text { финансирования }\end{array}$ & $\begin{array}{l}O D_{i}^{j}=\frac{d_{j}^{i} \text { факт }}{d_{j \text { пл }}^{i}} \\
\text { где } O D^{j}-\text { оценка степени выполнения плана финансирования меро- } \\
\text { приятий по ј-му факторному показателю для достижения і-го резуль- } \\
\text { тирующего показателя; } d_{j}^{i} \text { факт }, d_{j \text { нач }}^{i}-\text { фактический и плановый } \\
\text { объемы финансовых средств, направленных на выполнение ј-го } \\
\text { факторного показателя по і-му результирующего показателю }\end{array}$ \\
\hline $\begin{array}{l}\text { Степень использования } \\
\text { финансовых средств }\end{array}$ & $I D_{i}=\sum_{i} W_{j}^{i} * O D_{j}^{i}$ \\
\hline
\end{tabular}

Источник: разработка автора

Такая оценка позволит графически интерпретировать полученные данные, как показано на рисунке 3, на котором рассмотрены процессы реализации стратегии за период, равный шагу измерений стратегического планирования. Заметим, что построение методики определения коэффициентов важности имеет немаловажное значение и требует отдельного рассмотрения. 


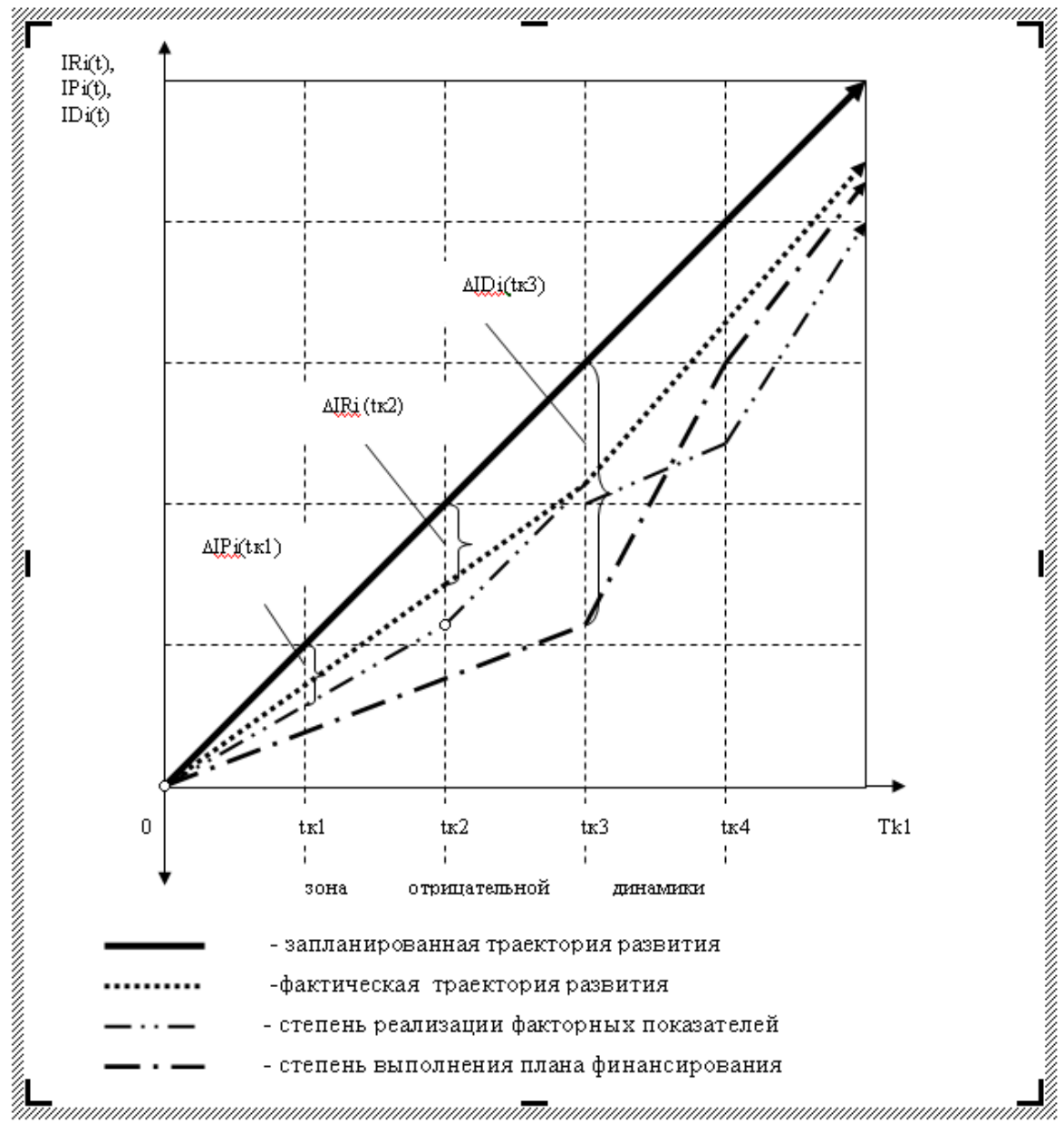

Рисунок 3 - Графическая интерпретация степени выполнения показателей Источник: разработка автора

Сопоставление степени выполнения различных показателей дает возможность разработать предложения по достижению запланированной траектории, либо предложения по корректировке самой траектории развития. Предлагаемый алгоритм принятия решений (рисунок 4) учитывает величину отклонений фактических показателей от запланированных. Если их амплитуда не более 0,025 - ситуация рассматривается как зона нормального режима работы; амплитуда отклонений в диапазоне от 0,025 до 0,05 также располагается в зоне естественных отклонений, если же отклонения более 0,05 - необходимы корректирующие действия, адекватные амплитуде отклонений [3, с. 19].

Возникающую в процессе оценки степени реализации показателей проблему различной их важности для достижения поставленных целей предлагается обосновать результатами экспертного опроса специалистов. Обоснование коэффициентов важности, как правило, проводится в два этапа: а) измерение (получение данных от экспертов); б) обработка данных формальными математическими методами/ 


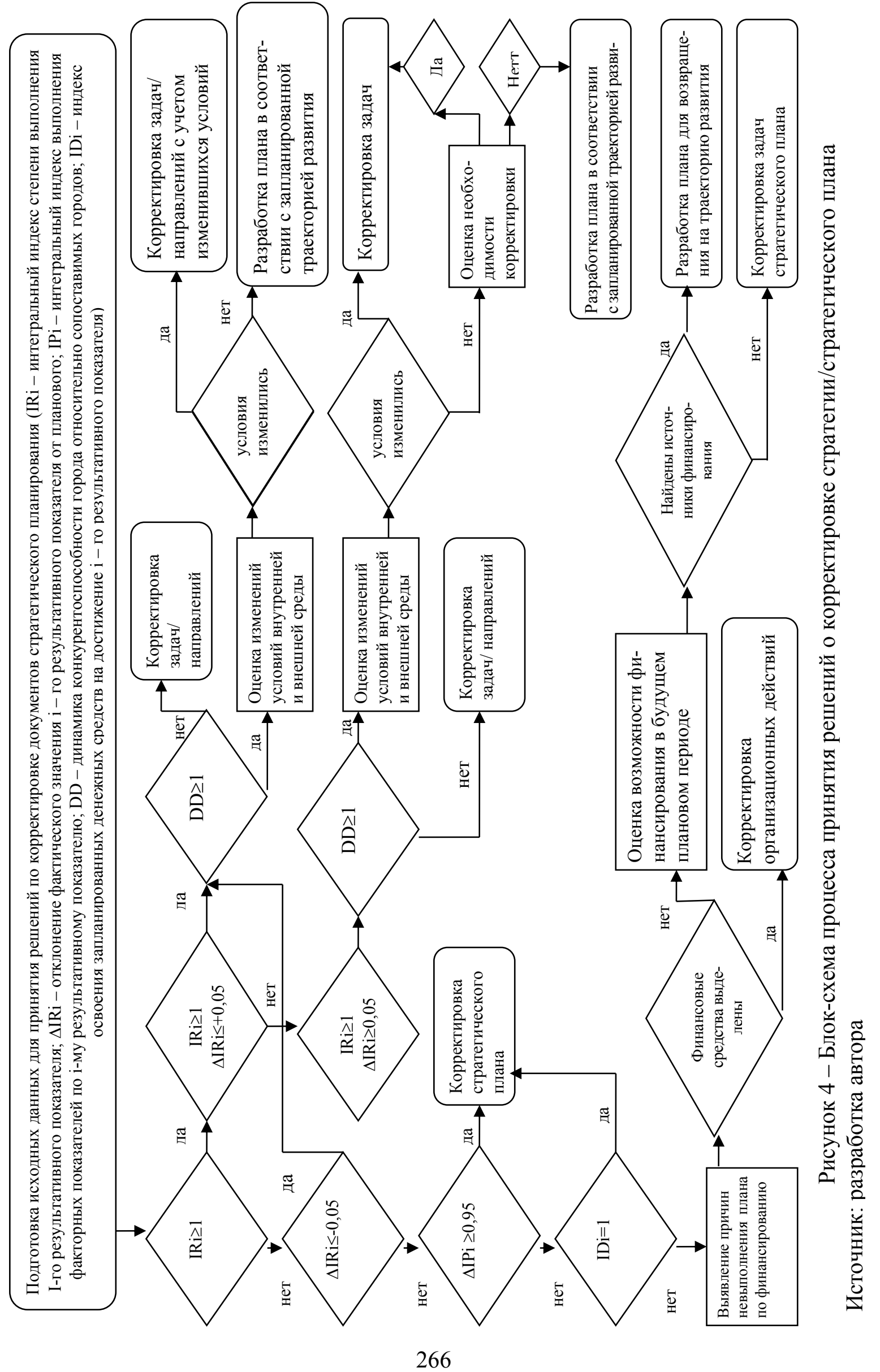


В результате этого процесса каждому $j$-му фактору ставится в соответствие неотрицательное число $W_{j}, j=\overline{1, m}$, отражающее относительную значимость фактора. При этом коэффициенты относительной важности факторов удовлетворяют условию $0<W_{j}<1$, при этом:

$$
\sum_{j=1}^{m} W_{j}=1
$$

где $\mathrm{m}$ - количество оцениваемых факторов.

Нами предлагается использовать для решения данной задачи метод собственных значений Т. Саати [4], основанный на выполнении экспертами парных сравнений оцениваемых факторов и дальнейшей обработке данной информации. Обоснование критериев относительной важности показателей проводится в следующей последовательности:

Разработка опросных листов. Чтобы использовать метод Т. Саати, необходимо получить сведения в форме матриц парных сравнений:

$$
O Z_{j g}^{i}=\left\|\begin{array}{ccccc}
O Z_{11}^{i} & \ldots & O Z_{1 g} & \ldots & O Z_{1 j}^{i} \\
O Z_{j 1}^{i} & \ldots & O Z_{j g}^{i} & \ldots & O Z_{j m}^{i} \\
O Z_{m 1}^{i} & \ldots & O Z_{m g}^{i} & \ldots & O Z_{m n}^{i}
\end{array}\right\|, \quad I=\overline{1, n}
$$

где I $=\overline{1, n}$ множество экспертов; $O Z_{j g}^{i}$ - результат сравнения ј-го фактора с g-ым, полученный і-ым экспертом.

Для перечня показателей, составленного в соответствии с иерархической моделью документов системы стратегического планирования повышения конкурентоспособности, разрабатываются листы экспертного опроса (таблица 3 ).

\begin{tabular}{|c|c|c|c|c|}
\hline Условное наименование & 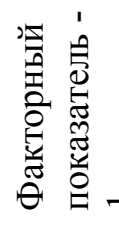 & 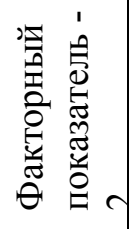 & 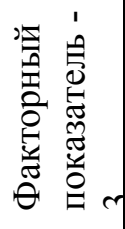 & 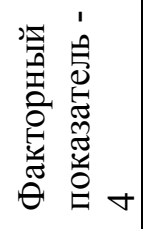 \\
\hline Факторный показатель - 1 & 1 & & & \\
\hline Факторный показатель - 1 & & 1 & & \\
\hline Факторный показатель - 1 & & & 1 & \\
\hline Факторный показатель - 1 & & & & 1 \\
\hline
\end{tabular}

Таблица 3 - Лист экспертного опроса

Источник: разработка автора

Оценки $O Z_{j g}^{i}$ назначаются в соответствии с вербально-числовой шкалой значений как показано в таблице 3.

Таблица 4 - Вербально-числовая шкала оценки попарного сравнения

\begin{tabular}{|c|l|}
\hline Числовая оценка & \multicolumn{1}{c|}{ Характеристика попарного сравнения } \\
\hline 1 & j-ый и g-ый факторы имеют равную важность \\
\hline 3 & j-ый фактор немного важнее g-го фактора \\
\hline 5 & j-ый фактор важнее g-го фактора \\
\hline 7 & j-ый фактор значительно важнее g-го фактора \\
\hline 9 & j-ый фактор абсолютно важнее g-го фактора \\
\hline
\end{tabular}

Источник: разработка автора на основе [4]. 
2. Разработанные листы заполняются экспертами, причем заполняется только верхняя от диагонали часть матрицы, заполнение нижней части проводится на основе использования условия взаимного дополнения оценок:

$$
O Z_{j g}=\frac{1}{O Z_{g i}}
$$

3. В отношении строк матрицы, заполненных каждым экспертом, определяется произведение их М элементов (ячеек), из которого извлекается корень степени $\mathrm{m}$, т. е. рассчитывается величина:

$$
O Z_{S}^{i}=\sqrt[m]{O Z_{11}^{i} \cdot O Z_{1 g}^{i} \cdot \ldots \cdot O Z_{1 m}^{i}}
$$

4. Рассчитанные величины суммируются, т.е. в отношении матриц, заполненных каждым экспертом, определяется величина:

$$
O Z_{S}^{i}=\sum_{j=1}^{m} O Z_{j}^{i}
$$

На основе нормализации полученных чисел определяются коэффициенты значимости с позиции каждого эксперта:

$$
W_{j}^{i}=\frac{o Z_{j}^{i}}{o Z_{S}^{i}}
$$

где $W_{j}^{i}$ - коэффициент важности ј-го фактора с позиции і-го эксперта.

5. Проверяется правильность расчетов путем суммирования полученных коэффициентов значимости по каждому фактору в целях соблюдения выполнения условия (формула 1).

6. Определяются коэффициенты важности факторов как среднее арифметическое коэффициентов важности, полученных на основе обработки результатов экспертного опроса всех экспертов:

$$
W_{j}=\frac{1}{n} \cdot \sum_{i=1}^{n} W_{j}^{i},
$$

Использование метода Т. Саати позволяет: а) свести к минимуму затруднения экспертов в процессе выражения своих суждений; б) существенно уменьшить влияние ошибок, допущенных экспертами при осуществлении попарного сравнения факторов, на этапе обработки данных.

\section{ВЫВОДЫ}

Таким образом, при использовании предлагаемого методического обеспечения возникает возможность оценить: а) качество стратегического прогнозирования и планирования, выбора стратегических направлений развития и соответствующих задач для их реализации; б) качество текущего планирования, действенность предпринимаемых для решения поставленных задач мер, достаточность финансовых средств, выделяемых на решение поставленных задач, уровень компетентности лиц, ответственных за реализацию запланированных мероприятий; в) необходимость корректирующих действий. От- 
метим, что данное методическое обеспечение применимо, в том числе, к оценке реализации программных документов различных уровней (национального, регионального, локального), направленности и горизонта планирования.

\section{СПИСОК ИСПОЛЬЗОВАННЫХ ИСТОЧНИКОВ}

1. Горева, М. А. Маркетинговая стратегия гостеприимного города: автореферат диссертации на соиск. степ. канд. эк. наук по спец. 08.00.05 - М., 2011. - 25 с.

2. Меньщикова, В. И. Стратегия социально-экономического развития в системе управления регионом / В. И. Меньщикова // Вестник ТГУ. - Тамбов, 2009. - с. 77-87

3. Высоцкий, О. А. Прозрачное управление в системе обеспечение устойчивого развития предприятия / О. А. Высоцкий. - Мн: Право и экономика, 2014. - 54 с. - (серия «Высшая школа бизнеса)

4. Никул, Е. С. Алгоритм анализа матриц парных сравнений с помощью вычисления векторов приоритетов / Е. С. Никул [Электронный ресурс]. - Режим доступа: https:// cyberleninka.ru/article/n/algoritm-analiza-matrits-parnyh-sravneniy-s-pomoschyuvychisleniya-vektorov-prioritetov. - Дата доступа: 17.10.2017.

\section{REFERENCES}

1. Goreva, M. A. Marketingovaya strategiya gostepriimnogo goroda: avtoreferat dissertatsii na soisk. step. kand. ek. nauk po spets. 08.00.05 - M., 2011. - $25 \mathrm{~s}$.

2. Menschikova, V. I. Strategiya sotsialno-ekonomicheskogo razvitiya v sicteme upravleniya regionom / Vestnik TGU. - Tambov, 2009. - s. 77-87

3. Vyisotskiy, O. A. Prozrachnoe upravlenie v sisteme obespechenie ustoychivogo razvitiya predpriyatiya / O. A. Vyisotskiy. - Mnyu: Pravo i ekonomika, 2014. - 54 s. - (seriya «Vyisshaya shkola biznesa)

4. Nikul, E. S. Algoritm analiza matrits parnyih sravneniy s pomoschyu vyichisleniya vektorov prioritetov [Elektronnyiy resurs]. - Rezhim dostupa : https://cyberleninka.ru/ article/n/algoritm-analiza-matrits-parnyh-sravneniy-s-pomoschyu-vychisleniya-vektorov-priorite tov. - Data dostupa: 17.10.2017.

Статья поступила в редакциию 28 февраля 2018 года. 